\title{
6 \\ Retarding the growth of the Rosensweig instability unveils a new scaling regime
}

\author{
Adrian Lange, ${ }^{1, *}$ Christian Gollwitzer, ${ }^{2, \dagger}$ Robin Maretzki, ${ }^{2}$ Ingo Rehberg, ${ }^{2, \ddagger}$ and Reinhard Richter ${ }^{2, \S}$ \\ ${ }^{1}$ TU Dresden, Institute of Fluid Mechanics, D-01062 Dresden, Germany \\ ${ }^{2}$ Experimentalphysik V, Universität Bayreuth, D-95440 Bayreuth, Germany
}

(Received 25 January 2016; published 5 April 2016)

\begin{abstract}
Using a highly viscous magnetic fluid, the dynamics in the aftermath of the Rosensweig instability can be slowed down by more than 2000 times. In this way we expand the regime where the growth rate is predicted to scale linearly with the bifurcation parameter by six orders of magnitude, while this regime is tiny for standard ferrofluids and cannot be resolved experimentally there. We measure the growth of the pattern by means of a two-dimensional imaging technique, and find that the slopes of the growth and decay rates are not the same-a qualitative discrepancy with respect to the theoretical predictions. We solve this discrepancy by taking into account a viscosity which is assumed to be different for the growth and decay. This may be a consequence of the measured shear thinning of the ferrofluid.
\end{abstract}

DOI: 10.1103/PhysRevE.93.043106

\section{INTRODUCTION}

The "pitch-drop experiment" [1], which received the Ig Nobel Prize in physics 2005, has brought to attention that a fast process like drop formation [2,3] can be retarded considerably if instead of a standard liquid like water-it has a viscosity of $10^{-3} \mathrm{Pas}$ at $20^{\circ} \mathrm{C}-\mathrm{a}$ material like pitch, with a viscosity around $10^{8} \mathrm{~Pa}$ s, is selected. The funnel was filled in 1930 [4]; in 2014, "Finally the ninth Pitch Drop has fallen from the world's longest running lab experiment" [5], and the tenth is awaited within the next 14 years. Here the question arises whether those high viscosities may give access to so far unresolved phenomena.

In the following we are investigating this question for the case of the well known Rosensweig or normal field instability [8]. It is observed in a horizontal layer of magnetic fluid (MF) [9] with a free surface, when a critical value $B_{c}$ of the vertically oriented magnetic induction is surpassed. Figure 1 presents a photo of the final hexagonal arrangement of static liquid peaks. Besides the threshold, beyond which the instability occurs, two quantities characterizing the emerging pattern have been in the focus of various studies: the critical wave number of the peaks and the corresponding growth rate. Both are strongly influenced by the viscosity of the magnetic fluid.

That essential role of the viscosity for the dynamics of the pattern formation is reflected in the course of the analyses devoted to the Rosensweig instability. For an inviscid magnetic fluid (the dynamic viscosity $\eta$ is zero) and an infinitely deep container, Cowley and Rosensweig [8] provide a linear stability analysis already in the very first description of the normal field instability to find the critical threshold $B_{\mathrm{c}}$ and the critical wave number $k_{\mathrm{c}}$. This approach was extended later by Salin [10] to fluids with nonzero viscosity, where the growth rate depends on $\eta$, and to a finite depth of the container by Weilepp and Brand [11]. First experimental investigations on the growth of the pattern were provided by

\footnotetext{
*Adrian.Lange@tu-dresden.de

†hristian.Gollwitzer@ptb.de

†Ingo.Rehberg@uni-bayreuth.de

${ }^{\S}$ Reinhard.Richter@uni-bayreuth.de
}

Lange et al. [12,13], who also derived the growth rate for the case of a viscous magnetic fluid and an arbitrary layer thickness $h$. This theoretical analysis was later extended to the case of a nonlinear magnetization curve $M(H)$ by Knieling et al. [14].

Whereas so far the growth rate of the emerging Rosensweig pattern has been measured utilizing ferrofluids with $\eta=$ $4.2 \times 10^{-3} \mathrm{~Pa} \mathrm{~s}[12,14]$ and $5.2 \times 10^{-2} \mathrm{~Pa} \mathrm{~s}[14]$, we are tackling here the growth process in a ferrofluid which is a thousand times more viscous than the first one. Such a ferrofluid is being created by cooling a commercially available viscous ferrofluid (APG E32 from Ferrotec Co. ) down to $10^{\circ} \mathrm{C}$. The ferrofluid has now a viscosity of $(4.48 \pm 0.1)$ Pa s. In such a cooled Rosensweig (nicknamed Frozensweig) instability [15] the growth of the pattern takes 60 seconds and can be measured with high temporal resolution in the extended system using a two-dimensional $x$-ray imaging technique [16,17]. That technique provides the full surface topography, as opposed to the $7 \mathrm{kHz}$ fast but one-dimensional Hall-sensor array, which had to be utilized for the low viscosity ferrofluids [14]. The potential of the retarded instability was demonstrated before [18], when the coefficients of nonlinear amplitude equations were determined in this way. In addition a sequence of localized patches of Rosensweig pattern were uncovered most recently [19] with that technique.

Here we exploit a higher viscosity to investigate the linear growth rate in a regime that was hitherto not accessible. This expectation is based on a scaling analysis presented in Ref. [20]. For supercritical inductions larger than $\bar{v}^{2} / 6$ [the dimensionless kinematic viscosity $\bar{v}$ is defined in Eq. (3.6c) below] the behavior of the growth rate is characterized by a square-root dependence on those inductions, as confirmed in [14]. In contrast, for supercritical inductions smaller than $\bar{v}^{2} / 6$ the behavior of the growth rate is characterized by a linear dependence. In the present experiment we increase $\bar{v}^{2} / 6$ by six orders of magnitude due to the high viscosity of the ferrofluid APG E32 at $10^{\circ} \mathrm{C}$. Thus a new territory of linear scaling is open for exploration.

The outline of the paper is as follows: the experimental setup and the measurements are sketched next in Sec. II. The theoretical analysis is presented in Sec. III and subsequently compared with the experimental findings in Sec. IV. 


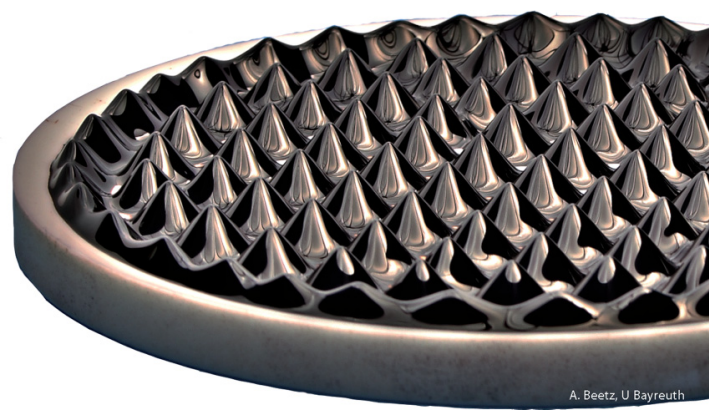

FIG. 1. Rosensweig peaks of the magnetic fluid EMG 909 at a supercritical induction $B>B_{\mathrm{c}}$ in a vessel with diameter of $120 \mathrm{~mm}$. The picture is taken from [6]. A movie showing the formation of Rosensweig patterns can be accessed at [7].

\section{EXPERIMENT}

In this section we describe the experimental setup (Sec. II A), the ferrofluid (Sec. II B), the protocol utilized for the measurements (Sec. II C), and the way the linear growth rate is extracted from the recorded data (Sec. II D).

\section{A. Experimental setup}

The experimental setup for the measurements of the surface topography consists of a custom made $\mathrm{x}$-ray apparatus described in detail before [16,17]. An x-ray point source emits radiation vertically from above through the container filled with the MF. Underneath the container, an X-ray camera records the radiation passing through the layer of MF. The intensity at each pixel of the detector is directly related to the height of the fluid above that pixel, as sketched in Fig. 2(a). Therefore, the full surface topography can be reconstructed after calibration $[16,17]$.

The container that holds the MF sample is depicted in Fig. 2(b). It is a regular octagon machined from aluminum with a side length of $77 \mathrm{~mm}$ and two concentric inner bores with a diameter of $140 \mathrm{~mm}$. These circular holes are carved from above and below, leaving only a thin base in the middle of the vessel with a thickness of $2 \mathrm{~mm}$. On top of the octagon, a circular aluminum lid is placed, which closes the hole from above, as shown in Fig. 2(b). Each side of the octagon is equipped with a thermoelectric element, QC-127-1.4-8.5MS from Quick-Ohm, as shown in Fig. 2(c). The elements are powered by a $1.2 \mathrm{~kW}$ Kepco KLP-20-120 power supply. The hot side of the Peltier elements is connected to water cooled heat exchangers. The temperature is measured at the bottom of the aluminum container with a Pt100 resistor. The temperature difference between the center and the edge of the bottom plate does not exceed $0.1 \mathrm{~K}$ at the temperature $\theta=10.0^{\circ} \mathrm{C}$ measured at the edge of the vessel. A closed loop control, realized using a computer and programmable interface devices, holds $\theta$ constant within $10 \mathrm{mK}$.

The container is surrounded by a Helmholtz pair of coils, thermally isolated from the vessel with a ring made from the flame resistant material FR-2. The size of the coils is adapted to the size of the vessel in order to introduce a "magnetic ramp" at the edge of the vessel. This technique, as described in more detail in Ref. [21], serves to minimize distortions

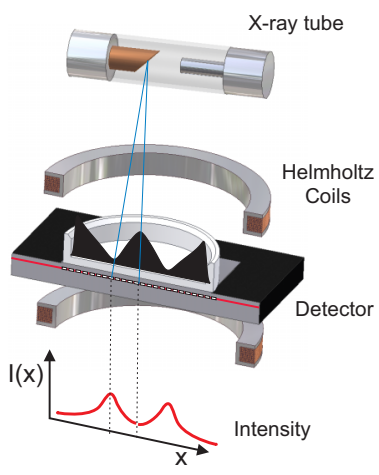

(a)

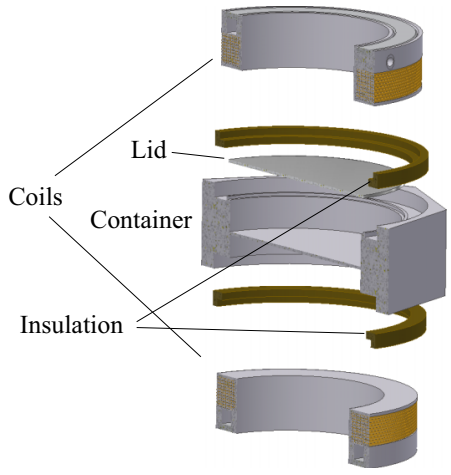

(b)

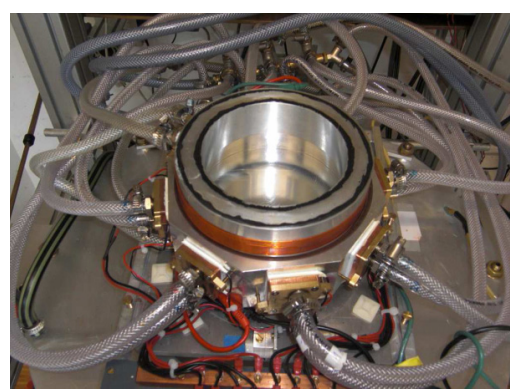

(c)

FIG. 2. Setup of the apparatus for dynamic measurements of the Rosensweig instability. (a) Sketch of the assembled setup of the xray machine. (b) Detail of the fragmented container with the coils generating the magnetic field. (c) Photo of the open container, the upper coil, and the water cooled peltier elements.

by partly compensating for the jump of the magnetization at the container edge. Filling the container to a height of $5 \mathrm{~mm}$ with ferrofluid enhances the magnetic induction in comparison with the empty coils for the same current $I$. Therefore $B(I)$ is measured immediately beneath the bottom of the container, at the central position, and serves as the control parameter in the following.

\section{B. Characterization of the ferrofluid}

The vessel is filled with the commercial magnetic fluid APG E32 from Ferrotec Co. up to a height of $5 \mathrm{~mm}$. The material parameters of this MF are listed in Table I. The density was measured using a DMA 4100 density meter from Anton Paar. The surface tension was measured using a commercial ring tensiometer (Lauda TE 1) and a pendant drop method (Dataphysics OCA 20). Both methods result in a surface tension of $\sigma=(31 \pm 0.5) \mathrm{mN} \mathrm{m}^{-1}$; but, when the liquid is allowed to rest for one day, $\sigma$ drops down $(25 \pm 0.5) \mathrm{mN} \mathrm{m}^{-1}$. This effect, which is not observed in similar but less viscous magnetic liquids like the one used in Ref. [17], gives a hint that the surfactants change the surface tension at least on a longer time scale, when the surface is changed. Since indeed the pattern formation experiments do change the surface during the measurements, the uncertainty of the surface tension is $\approx 5 \mathrm{mN} \mathrm{m}^{-1}$, as given in Table I.

a. Magnetization curve. The magnetization has been determined using a fluxmetric magnetometer (Lakeshore Model 
TABLE I. Material properties of the magnetic fluid APG E32 (Lot G090707A) from Ferrotec Co at $10^{\circ} \mathrm{C}$.

\begin{tabular}{|c|c|c|c|c|}
\hline Quantity & & Value & Error & Unit \\
\hline Density & $\rho$ & 1168.0 & \pm 1 & $\mathrm{~kg} \mathrm{~m}^{-3}$ \\
\hline Surface tension & $\sigma$ & 30.9 & \pm 5 & $\mathrm{mN} \mathrm{m}^{-1}$ \\
\hline Viscosity & $\eta$ & 4.48 & \pm 0.1 & Pas \\
\hline Saturation magnetization & $M_{\mathrm{S}}$ & 26.6 & \pm 0.8 & $\mathrm{kA} \mathrm{m}^{-1}$ \\
\hline Initial susceptibility & $\chi_{0}$ & 3.74 & \pm 0.005 & \\
\hline \multicolumn{5}{|l|}{ Fit of $M(H)$ with the model by Ref. [23] } \\
\hline Exponent of the $\Gamma$ distribution & $\alpha_{\Gamma}$ & 3.8 & \pm 1 & \\
\hline Typical diameter of the bare particles & $d_{0}$ & 1.7 & \pm 0.2 & $\mathrm{~nm}$ \\
\hline Volume fraction of the magnetic material & $\phi$ & 5.96 & \pm 0.2 & $\%$ \\
\hline \multicolumn{5}{|l|}{ Fit of $\eta(H)$ with the model by Ref. [24] } \\
\hline Mean diameter of the bare particle & $d_{\mathrm{m}}$ & 15 & & $\mathrm{~nm}$ \\
\hline Volume fraction of the magnetic material & $\phi$ & 21.4 & \pm 0.2 & $\%$ \\
\hline Critical induction for a semi-infinite layer [25] & $B_{\mathrm{c}, \text { theo, lin, } \infty}$ & 10.5 & \pm 0.1 & $\mathrm{mT}$ \\
\hline
\end{tabular}

480) constructed to deal with larger samples of high viscosity at a temperature of $\theta=20^{\circ} \mathrm{C}$ [22]. Figure 3 shows the data, which have been fitted by the modified mean field model of second order [23], marked by the dashed black line. For a comparison with the pattern formation experiments performed at $\theta=10^{\circ} \mathrm{C}$, the curve is extrapolated utilizing this model (blue line). The deviation between the curves is tiny, which was corroborated with a vibrating sample magnetometer (Lakeshore VSM 7404) at $\theta=20^{\circ} \mathrm{C}$ and $10^{\circ} \mathrm{C}$. Note that the VSM offers the advantage that it can be tempered, but has a lower resolution in comparison to the fluxmetric device because of the smaller sample volume.

To take into account the nonlinear $M(H)$, an effective susceptibility $\bar{\chi}_{\mathrm{H}}$ is defined by a geometric mean

$$
1+\bar{\chi}_{\mathrm{H}}=\sqrt{\left(1+\chi_{\mathrm{ta}}\right)\left(1+\chi_{\mathrm{ch}}\right)},
$$

with the tangent susceptibility $\chi_{\mathrm{ta}}=\partial M / \partial H$ and the chord susceptibility $\chi_{\mathrm{ch}}=M / H$ [26]. For any field $H$ the effective susceptibility $\bar{\chi}_{\mathrm{H}}$ can be evaluated, when the magnetization $M(H)$ curve is known.

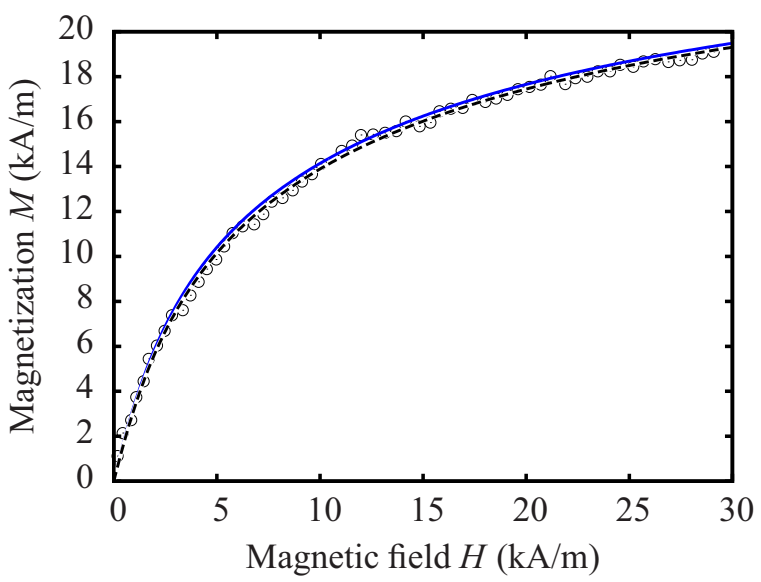

FIG. 3. Magnetization curve of the magnetic fluid APG E32 measured with a fluxmetric magnetometer. The symbols show the measured data at $\theta=20^{\circ} \mathrm{C}$. The black dashed line is a fit with the model from Ref. [23]. The blue solid line marks an extrapolation to $\theta=10^{\circ} \mathrm{C}$ according to this model. b. Viscosity. The viscosity $\eta$ deserves special attention for the experiments, as it influences the time scale of the pattern formation. It has been measured in a temperature range of $-5^{\circ} \mathrm{C} \leqslant \theta \leqslant 20^{\circ} \mathrm{C}$, using a commercial rheometer (MCR 301, Anton Paar) with a shear cell featuring a cone-plate geometry. At room temperature, the magnetic fluid with a viscosity of $\eta=2 \mathrm{~Pa}$ s is 2000 times more viscous than water. The value of $\eta$ can be increased by factor of 9 when the liquid is cooled to $-5^{\circ} \mathrm{C}$. The temperature-dependent viscosity data can be nicely fitted with the well-known Vogel-Fulcher law [27]

$$
\eta=\eta_{0} \exp \left(\frac{\psi}{\theta-\theta_{0}}\right)
$$

with $\eta_{0}=0.48 \mathrm{mPas}, \psi=1074 \mathrm{~K}$, and $\theta_{0}=-107.5^{\circ} \mathrm{C}$, as described in detail in Ref. [18]. For the present measurements, we chose a temperature of $\theta=10^{\circ} \mathrm{C}$, where the viscosity amounts to $\eta=4.48 \mathrm{~Pa}$ s according to Eq. (2.2).

c. Magnetoviscosity. The growth and decay of ferrofluidic spikes takes place in a magnetic field, which is known to alter the viscosity. Furnishing the rheometer with the magnetorheological device MRD 170-1T from Anton Paar, we exemplarily measure the magnetoviscous behavior for a shear rate of $\dot{\gamma}=1 \mathrm{~s}^{-1}$. We use a plate-plate configuration with a gap of $300 \mu \mathrm{m}$, where the upper plate has a diameter of $20 \mathrm{~mm}$. Figure 4 displays the measured data together with a fit by

$$
\eta(\alpha)=\left.\eta\right|_{\alpha=0}+\eta_{\mathrm{r}}(\alpha)=\left.\eta\right|_{\alpha=0}+\frac{3}{2} \Phi_{\mathrm{h}} \eta \frac{\alpha-\tanh \alpha}{\alpha+\tanh \alpha}\left\langle\sin ^{2} \beta\right\rangle,
$$

which describes the magnetoviscosity according to Shliomis [24]. Here $\alpha=\mu_{0} M_{\mathrm{d}} V H_{\mathrm{i}} /\left(k_{\mathrm{B}} T\right)$, denotes the ratio between the magnetic energy of the dipole in the field $H_{\mathrm{i}}$ and the thermal energy $k_{\mathrm{B}} T$, where $M_{\mathrm{d}}=446 \mathrm{kA} / \mathrm{m}$ is the domain magnetization of saturated magnetite [9], and $V$ the magnetic active volume. Moreover $\left.\eta\right|_{\alpha=0}$ captures the viscosity without a magnetic field, $\eta_{\mathrm{r}}$ is the additional rotational viscosity due to the presence of the magnetic field $\vec{H}_{\mathrm{i}}$ in the ferrofluid, and $\Phi_{\mathrm{h}}$ is the hydrodynamic volume fraction of the magnetite particles. The brackets $\langle\cdots\rangle$ indicate a spatial average over the enclosed quantity. Note that in the case of Fig. 4 the angle 


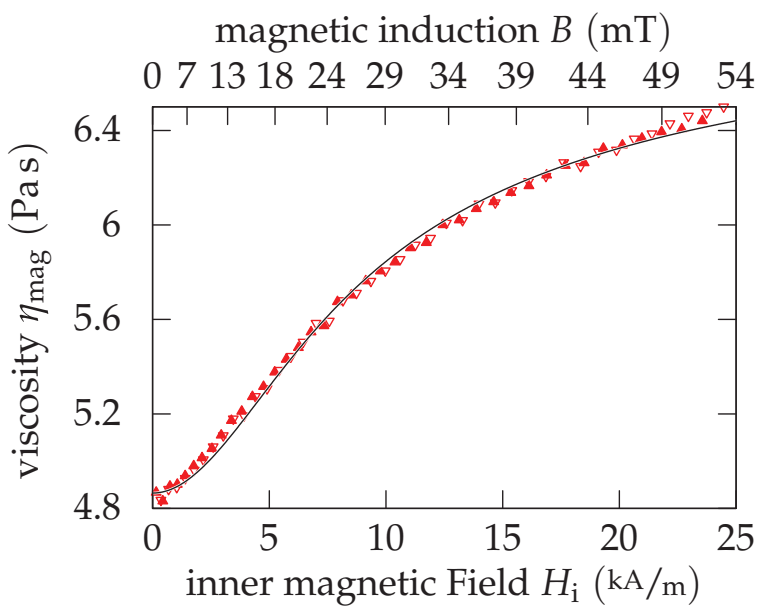

FIG. 4. The magnetoviscosity of the ferrofluid APG E32 versus the inner field $H_{\mathrm{i}}$ for a shear rate of $\dot{\gamma}=1 \mathrm{~s}^{-1}$. The $\boldsymbol{\Delta}(\nabla)$ mark measurements for increasing (decreasing) $H_{\mathrm{i}}$ and the solid line is a fit by Eq. (2.3). The upper abscissa displays the applied magnetic induction $B$ measured in the air gap beneath the magnetorheological cell.

$\beta$ between $\vec{H}_{\mathrm{i}}$ and the vorticity of the flow is $90^{\circ}$. For the fit the internal field was obtained via solving $H_{\mathrm{i}}=B / \mu_{0}-D M\left(H_{\mathrm{i}}\right)$, assuming a demagnetization factor of $D=1$. The fit yields a hydrodynamic volume fraction of $\Phi_{\mathrm{h}}=43.5 \pm 0.1 \%$ and $\alpha / H_{\mathrm{i}}=256 \pm 2 \times 10^{-6} \mathrm{~m} / \mathrm{A}$. From $V$ one estimates a mean diameter of $d_{\mathrm{m}}=15 \mathrm{~nm}$ for the magnetic particles. This is almost a factor of 10 larger than $d_{0}=1.7 \mathrm{~nm}$ obtained from the magnetization curve (cf. Table I). Assuming a spherical layer of oleic acid molecules of thickness $\delta=2 \mathrm{~nm}$ around the magnetic particles [9], the volume fraction of the magnetic active material is $\Phi=\Phi_{\mathrm{h}}\left(1+2 \delta / d_{\mathrm{m}}\right)^{-3}=21.4 \%$. This is more than three times larger than the value obtained via the magnetization curve (cf. Table I). The elevated values of $d$ and $\Phi$ may be a consequence of magnetic agglomerates, which are not taken into account by Eq. (2.3).

To test the flow behavior of the ferrofluid, the viscosity was measured versus the shear rate for three exemplary magnetic inductions, as presented in Fig. 5. All curves exhibit a decay of the viscosity for increasing $\dot{\gamma}$, i.e., shear thinning which is typical for dispersions [28]. For a quantitative description of this effect the measured data are fitted by the Sisko equation [29]

$$
\eta(\dot{\gamma})=k \dot{\gamma}^{n-1}+\eta_{0}
$$

adapted to the limit $\dot{\gamma} \rightarrow 0 \mathrm{~s}^{-1}$, where $\eta \rightarrow \eta_{0}$. Moreover $k$ denotes a factor and $n$ a scaling exponent. Table II displays the fitting parameters obtained for the three inductions. Under

TABLE II. The parameters obtained by fitting Eq. (2.4) to the experimental data.

\begin{tabular}{lccc}
\hline \hline$B(\mathrm{mT})$ & $k\left(\mathrm{~Pa} \mathrm{~s}^{(2-\mathrm{n})}\right)$ & $n$ & $\eta_{0}(\mathrm{~Pa} \mathrm{~s})$ \\
\hline 0 & $-0.015 \pm 0.001$ & $1.62 \pm 0.02$ & $4.826 \pm 0.002$ \\
11.4 & $-0.035 \pm 0.001$ & $1.559 \pm 0.006$ & $5.107 \pm 0.002$ \\
114 & $-0.319 \pm 0.001$ & $1.346 \pm 0.004$ & $7.328 \pm 0.009$ \\
\hline \hline
\end{tabular}

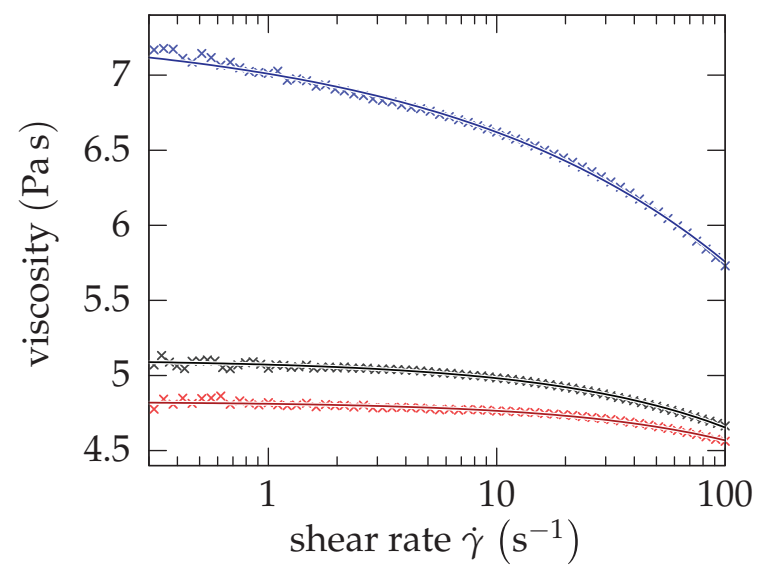

FIG. 5. The viscosity of the magnetic fluid APG E32 versus the applied shear rate $\dot{\gamma}$ for $B=0 \mathrm{mT}$ (red), $11.4 \mathrm{mT}$ (black), and $114 \mathrm{mT}$ (blue). The crosses mark the measured data (for clarity only every fifth data point is shown), whereas the solid lines display fits by Eq. (2.4).

increase of $B$ most prominently the factor $k$ is varying. For $B=0 \mathrm{mT}, k$ is tiny and we have an almost Newtonian liquid. The factor $k$ doubles at $B=11.4 \mathrm{mT}$, and eventually enlarges by a factor of 10 at the tenfold value of $B=114 \mathrm{mT}$. At the same time $\eta_{0}$ does not even double. This quantitative description is in agreement with the increasing decay of the curves in Fig. 5. The deepening of shear thinning with $B$ has been attributed to the formation of chains and agglomerates of magnetic particles in the field and their subsequent destruction under shear. Chains have been uncovered by transmission electron microscopy [30,31], and their destruction has been studied in magnetorheology [32]. For a review see, e.g., Ref. [33].

We conclude this topic by noting that the fit of the magnetoviscous behavior as well as the shear thinning indicate that agglomerates of magnetic particles are emerging in the field. In this way the faint non-Newtonian behavior of the suspension which is already present at zero induction may be enhanced considerably in the field and may cause unexpected dynamics.

\section{Measurement protocol}

Figure 6 displays the measurement protocol on the basis of the bifurcation diagram, measured in Ref. [18]. The static pattern amplitude of the Rosensweig instability in our fluid is indicated by the red line. When the system is set onto an arbitrary initial point $\left(B_{\text {ini }}, A_{\text {ini }}\right)$ in this diagram, and the magnetic induction $B$ is kept constant, the amplitude $A$ increases or decreases monotonically, until the system reaches the stable equilibrium (solid red line). The direction of the change of $A$ depends on the region where $\left(B_{\text {ini }}, A_{\text {ini }}\right)$ is situated: in regions I and III in Fig. $6 A$ increases, and in regions II and IV the amplitude decreases with time.

In order to push the system to an arbitrary initial location ( $B_{\text {ini }}, A_{\text {ini }}$ ), a three-step measurement protocol is employed. The first step (path $\uparrow 1$ ) is always a relaxation of the pattern in region $\mathrm{I}$ at the overcritical induction $B_{1}=11.45 \mathrm{mT}$ for $\tau_{1}=60 \mathrm{~s}$, to reach the high amplitude of $A_{\text {high }}=2.98 \mathrm{~mm}$ at that point. The corresponding pattern is shown in Fig. 7. 


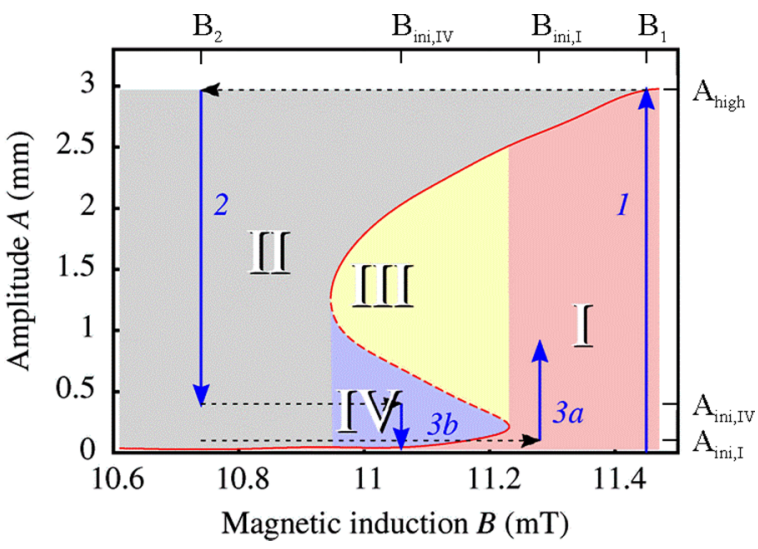

FIG. 6. The three-step measurement protocol for the growth and relaxation measurements. Dotted arrows indicate jumps of the magnetic inductions. Blue arrows denote the path of the system during the growth or relaxation phase. Note the different axis labels at the left and at the right, as well as at the bottom and at the top. A movie of such a process is available at [18].

Then the magnetic induction is quickly reduced to the value $B_{2}=10.74 \mathrm{mT}$, and the resulting dynamics is observed (path $\downarrow 2$ ), until the desired starting amplitude $\left(A_{\text {ini,II }}, A_{\text {ini,IV }}\right.$, or $\left.A_{\text {ini, }}\right)$ is reached after a period $\tau_{2}$. To start with this pattern at arbitrary inductions in the regimes II, IV, and I the induction is then quickly raised to the desired value $B_{\text {ini }}$. Then we record the pattern evolution along the path $\uparrow 3 \mathrm{a}$ or $\downarrow 3 \mathrm{~b}$ in regions II, IV, and I, respectively.

We use this detour instead of directly switching the magnetic induction from zero to $B_{\text {ini }}$ in order to establish the identical pattern in all regions. Coming from a perfectly flat surface, the pattern would have additional degrees of freedom; e.g., it could amplify any local disturbance, resulting in a propagating wave front on the liquid surface [21,34]. The emerging hexagonal pattern would comprise point defects or different orientations of the wave vectors [34,35]. When we take the detour by the paths $\uparrow 1$ and $\downarrow 2$, we seed a regular hexagonal pattern at $\left(B_{1}, A_{\text {high }}\right)$, and the evolving pattern is likely to be of the same regularity.

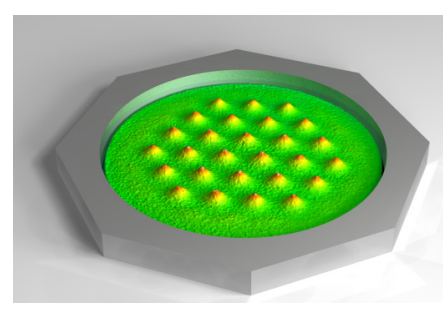

(a)

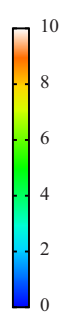

$(\mathrm{nm})$

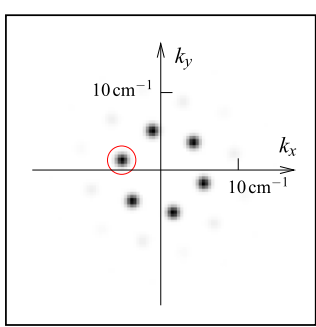

(b)
FIG. 7. The final pattern at $B_{1}=11.45 \mathrm{mT}$. Panel (a) displays a reconstruction of the surface in real space. The outer dimension of the container is not to scale. The color code gives the height of the liquid surface above ground in $\mathrm{mm}$. The pattern amplitude is determined from the corresponding power spectrum shown in (b) by the total power in the encircled mode. The data are taken from Ref. [18].

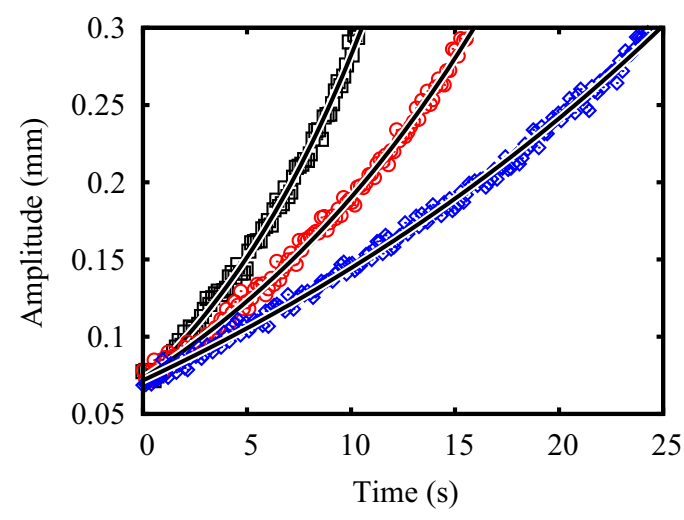

FIG. 8. Three examples for the growth of the pattern amplitude in region $\mathrm{I}$. The initial state was prepared with the sequence $B_{2}=$ $10.743 \mathrm{mT}, \tau_{2}=20.000 \mathrm{~s}$. The three curves show the measurements after switching to $B_{3}=11.455 \mathrm{mT}(\square), 11.376 \mathrm{mT}(\odot)$, and $11.323 \mathrm{mT}(\diamond)$. The solid black lines denote fits by Eq. (2.5).

\section{Extraction of the growth rate}

Next we describe the extraction of the growth rate from the recorded sequence of $x$-ray frames along the path $\uparrow 3 \mathrm{a}$ or $\downarrow 3 \mathrm{~b}$. From each $x$-ray frame the surface topography is reconstructed following the procedure described in Ref. [17]. As an example, Fig. 7(a) displays the resulting surface topography at $\left(B_{1}, A_{\text {high }}\right)$. The amplitude of the pattern is determined in Fourier space, as sketched in Fig. 7(b). We use a circularly symmetric Hamming window with a radius of $46 \mathrm{~mm}$ [18]. The total power in one of the modes, as marked in Fig. 7(b) by a red circle, is used to compute the amplitude of the pattern [18].

Figure 8 shows three exemplary curves for the growth of the pattern amplitude $A$. With increasing induction $B_{3}$ (from $\diamond$ via $\odot$ to $\square$ ) the growth increases; likewise Fig. 9 presents three examples of the decay of $A$, where $B_{3}$ denotes the initial induction $B_{\text {ini }}$ after the three steps of the detour procedure. Remarkably $A$ does not relax to zero, but to a small offset of $A_{\text {const }}$ which linearly increases from $32 \mu \mathrm{m}$ at $10.7 \mathrm{mT}$ to $34 \mu \mathrm{m}$ at $10.9 \mathrm{mT}$.

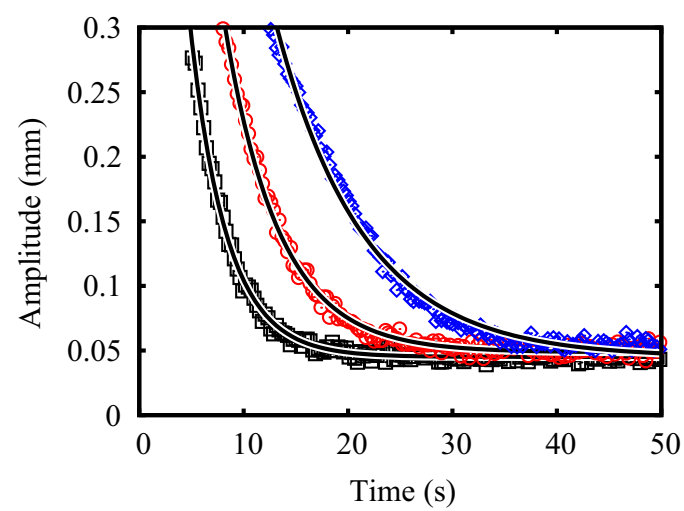

FIG. 9. Three examples for the decay of the pattern amplitude. The initial state was prepared with the sequence $B_{2}=10.743 \mathrm{mT}$, $\tau_{2}=10.000 \mathrm{~s}$. The three curves show the measurements after switching to $B_{3}=10.888 \mathrm{mT}(\square), 11.020 \mathrm{mT}(\odot)$, and $11.059 \mathrm{mT}$ $(\diamond)$. The solid black lines denote fits by Eq. (2.5). 


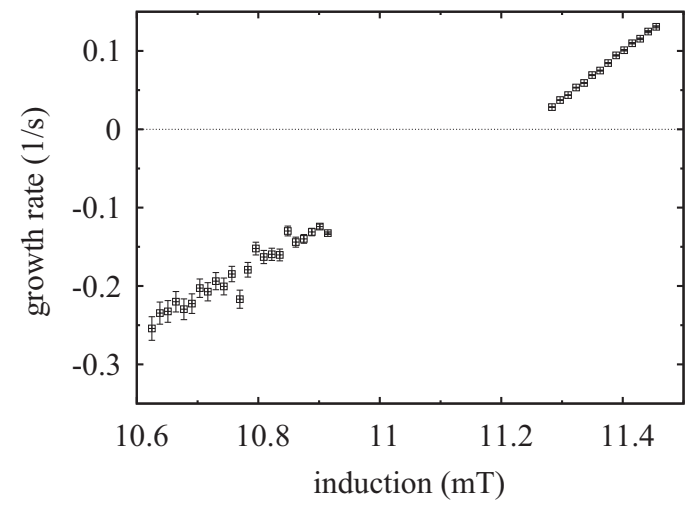

FIG. 10. The measured linear growth and decay rates $\omega_{2}$ of the pattern amplitude as a function of the magnetic induction $B$.

A possible explanation is imperfections induced by the lateral container wall, as observed before; see, e.g., Refs. [17,36]. In the present setup special precautions were taken by means of a "magnetic ramp" [21] to minimize such finite size effects. However, for an experimental setup with finite aspect ratio they cannot be excluded. The fact that $A_{\text {const }}$ does increase only slightly with $B$ does not contradict this assumption, because the decay is investigated in the regime II well below $B_{\mathrm{c}}$. From Fig. 6 one clearly sees that an increase of the imperfection becomes prominent only in the hysteretic regime (IV).

A further explanation would be an inhomogeneous distribution of surfactants at the surface of the MF, which develops after the massive destruction of surface area, and which cannot be followed up by the diffusion of the surfactants on the surface and into the bulk liquid. A resulting spatial variation of $\sigma$ would lead to surface crests, somehow reminiscent of those observed in Marangoni convection.

A third explanation is an inhomogeneous distribution of magnetic particles due to magnetophoresis [37] taking place while the field is at $B_{1}$. We consider this less likely because of the enormous time scales of such a process at the large viscosity of the experimental fluid.

From each measured curve we extract the linear growth or decay rate by a least square fit with the function

$$
A(t)=A_{0} \exp \left(\omega_{2} t\right)+A_{\text {const }},
$$

which is taking into account the constant offset. We restrict the fit to the interval $A \in[0.0,0.3] \mathrm{mm}$, for which we assume that a linear description is still possible. This is corroborated by exemplary fits in Figs. 8 and 9 which are marked by solid black lines.

In Fig. 10 we present the extracted growth and decay rate $\omega_{2}$ versus the applied induction $B=B_{3}$. Because of the large statistical errors of $\omega_{2}$ we have refrained from plotting the decay rate in the hysteretic regime. The measured values show a monotonic relation with the applied induction and indicate that a critical value for the magnetic induction of about $11.2 \mathrm{mT}$ exists. Using the material parameters from Table I and an infinite layer thickness yields $B_{\mathrm{c}, \text { theo,lin, } \infty}=10.5 \mathrm{mT}$ [8].

\section{THEORY}

The experimental system, described in Sec. II, is modeled as a horizontally unbounded layer of an incompressible, nonconducting, and viscous magnetic fluid subjected to a magnetic field which is perpendicular to the plain, horizontal, and undisturbed surface. The fluid is bounded from below by the bottom of a container made of a magnetically insusceptible material and has a free surface with air above.

According to the linear stability analysis [38], the pattern amplitude $A$ can be described by an exponential growth, $A \sim \exp (-i \tilde{\omega} t)$, with an exponent $\tilde{\omega}=\omega_{1}+i \omega_{2}$, when $A$ is small. The real part of $-i \tilde{\omega}, \omega_{2}$, is called the growth rate and defines whether the disturbances will grow $\left(\omega_{2}>0\right)$ or decay $\left(\omega_{2}<0\right)$. The absolute value of the imaginary part of $-i \tilde{\omega}$, $\left|\omega_{1}\right|$, gives the angular frequency of the oscillations if it is different form zero [38].

The exponent $\tilde{\omega}$ follows from the dispersion relation given in Ref. [14] for a layer of MF with the finite depth $h$, a nonlinear magnetization curve $M(H)$, the surface tension $\sigma$, the density $\rho$, and the kinematic viscosity $\nu$ :

$$
\begin{aligned}
0= & \frac{v^{2}}{\tilde{k} \operatorname{coth}(\tilde{k} h)-k \operatorname{coth}(k h)}\left(\tilde{k}\left[4 k^{4}+\left(k^{2}+\tilde{k}^{2}\right)^{2}\right]\right. \\
& \times \operatorname{coth}(\tilde{k} h)-k\left[4 k^{2} \tilde{k}^{2}+\left(k^{2}+\tilde{k}^{2}\right)^{2}\right] \\
& \left.\times \tanh (k h)-\frac{4 k^{2} \tilde{k}\left(k^{2}+\tilde{k}^{2}\right)}{\cosh (k h) \sinh (\tilde{k} h)}\right) \\
& +\tanh (k h)\left(g k+\frac{\sigma}{\rho} k^{3}-\frac{\mu_{0}\left(1+\bar{\chi}_{\mathrm{H}}\right) M^{2}}{\rho} \Lambda(k h) k^{2}\right),
\end{aligned}
$$

where

$$
\Lambda(k h)=\frac{e^{k h\left(1+\bar{\chi}_{\mathrm{H}}\right) /\left(1+\chi_{\mathrm{ta}}\right)}\left(2+\bar{\chi}_{\mathrm{H}}\right)-\bar{\chi}_{\mathrm{H}} e^{-k h\left(1+\bar{\chi}_{\mathrm{H}}\right) /\left(1+\chi_{\mathrm{ta}}\right)}}{e^{k h\left(1+\bar{\chi}_{\mathrm{H}}\right) /\left(1+\chi_{\mathrm{ta}}\right)}\left(2+\bar{\chi}_{\mathrm{H}}\right)^{2}-\bar{\chi}_{\mathrm{H}}^{2} e^{-k h\left(1+\bar{\chi}_{\mathrm{H}}\right) /\left(1+\chi_{\mathrm{ta}}\right)}}
$$

and

$$
\tilde{k}=\sqrt{k^{2}-\frac{i \tilde{\omega}}{v}}
$$

The solutions for the dispersion relation in the case of a linear magnetization curve were revised in Ref. [39]. The solution space is rather complex, but the following conclusions can be drawn: for $k=k_{\mathrm{c}}, \tilde{\omega}$ is purely imaginary and the pattern grows or decays exponentially.

\section{A. Scaling laws for a nonlinear magnetization}

In the following we study the generic dependence of the maximal growth rate $\omega_{2, \mathrm{~m}}$ and the corresponding wave number $k_{\mathrm{m}}$ on the nonlinear magnetization of the fluid and its viscosity. The reason is that $\omega_{2, \mathrm{~m}}$ and $k_{\mathrm{m}}$ characterize the linearly most unstable pattern. The dispersion relation (3.1) for $\tilde{\omega}=i \omega_{2}$, and an infinitely thick layer, $h \rightarrow \infty$, [10]

$$
\begin{aligned}
\sqrt{1+\frac{\omega_{2}}{v k^{2}}}= & \left(1+\frac{\omega_{2}}{2 v k^{2}}\right)^{2}+\frac{1}{4 \rho v^{2} k^{4}}\left[\rho g k+\sigma k^{3}\right. \\
& \left.-\frac{\mu_{0}\left(1+\bar{\chi}_{\mathrm{H}}\right)}{\left(2+\bar{\chi}_{\mathrm{H}}\right)} M^{2} k^{2}\right]
\end{aligned}
$$


is written in dimensionless form (indicated by a bar)

$$
\sqrt{1+\frac{\bar{\omega}_{2}}{\bar{\nu} \bar{k}^{2}}}=\left(1+\frac{\bar{\omega}_{2}}{2 \bar{\nu} \bar{k}^{2}}\right)^{2}+\frac{\bar{k}+\bar{k}^{3}-2 \bar{k}^{2} \bar{M}^{2} \chi_{\mathrm{rel}}}{4 \bar{v}^{2} \bar{k}^{4}} .
$$

For this result, any length, the time, the kinematic viscosity, and the magnetization were rescaled to dimensionless quantities using

$$
\begin{aligned}
& \bar{l}=k_{\mathrm{c}, \infty} l=\sqrt{\frac{\rho g}{\sigma}} l, \\
& \bar{t}=\frac{t}{t_{\mathrm{c}}}=\frac{g^{3 / 4} \rho^{1 / 4}}{\sigma^{1 / 4}} t, \\
& \bar{v}=\frac{g^{1 / 4} \rho^{3 / 4}}{\sigma^{3 / 4}} v,
\end{aligned}
$$

and

$$
\bar{M}=\frac{M}{M_{\mathrm{c}, \infty}},
$$

where

$$
M_{\mathrm{c}, \infty}=\sqrt{\frac{2}{\mu_{0}}\left(\frac{2+\bar{\chi}_{\mathrm{H}_{\mathrm{c}}}}{1+\bar{\chi}_{\mathrm{H}_{\mathrm{c}}}}\right) \sqrt{\rho g \sigma}}
$$

gives the critical magnetization for a semi-infinite layer of MF.

To find a scaling law for the growth rate, we differentiate implicitly the dimensionless dispersion relation (3.5) with respect to $\bar{M}$; i.e., we determine the slope of the growth rate called $\Gamma$. By taking the limit $\bar{M}=1$ we find $\Gamma$ in the vicinity of the point of bifurcation,

$$
\Gamma=\left.\frac{\partial \bar{\omega}_{2}}{\partial \bar{M}}\right|_{\bar{M}=1}=\frac{2 \chi_{\mathrm{rel}}}{\bar{v}} .
$$

Inspecting Eq. (3.7) one sees that $\Gamma$ is independent of the wave number $\bar{k}$ and is a finite constant. Exploiting the latter and using that, at the point of bifurcation $\bar{\omega}_{2}(\bar{M}=1)=0$ holds, the following scaling law can be formulated:

$$
\bar{\omega}_{2}=\frac{2 \chi_{\mathrm{rel}}}{\bar{v}} \hat{M} .
$$

That linear dependence of $\bar{\omega}_{2}$ on $\hat{M}$ is universal and is depicted already in the measured growth rates presented in Fig. 10. Since $\Gamma$ scales with $1 / \bar{v}$, the slope of the growth rates goes to infinity in the limit of inviscid fluids. Moreover, for normal magnetic fluids with their rather low viscosity, the range of validity of Eq. (3.8) is bounded by $\bar{v}^{2} / 6$ which is very small; see the third column, third line in Table III. Therefore this scaling law is only of limited practical value.

For low viscosity fluids it holds that $\omega_{2} /\left(\nu k^{2}\right)=$ $\bar{\omega}_{2} /\left(\bar{v} \bar{k}^{2}\right) \gg 1$; see the third column, last line in Table III. With the latter inequality, Eq. (3.5) simplifies to

$$
\bar{\omega}_{2}^{2}=-\bar{k}-\bar{k}^{3}+2 \bar{k}^{2} \bar{M}^{2} \chi_{\mathrm{rel}},
$$

and one can now determine the slope of the square of the growth rate:

$$
\Gamma_{\text {low vis }}=\frac{\partial \bar{\omega}_{2}^{2}}{\partial \bar{M}}=4 \bar{k}^{2} \chi_{\text {rel }} \bar{M}
$$

TABLE III. Essential features of a high viscosity magnetic fluid like APG E32 and those of a low viscosity fluid like EMG 909 associated with Eq. (3.10).

\begin{tabular}{lcc}
\hline \hline Quantity & High viscosity MF & Low viscosity MF \\
\hline$v\left(\mathrm{~m}^{2} / \mathrm{s}\right)$ & $\simeq 3.8 \times 10^{-3}$ & $\simeq 4.2 \times 10^{-6}[14]$ \\
$\bar{v}$ & 18.4 & $\simeq 2.2 \times 10^{-2}[14]$ \\
$\bar{v}^{2} / 6$ & 56.4 & $\simeq 7.8 \times 10^{-5}$ \\
wave number & $k \simeq k_{\mathrm{c}, \infty}$ & $\simeq k_{\mathrm{c}, \infty}\left(1+\tilde{c}_{3} \hat{M}+\tilde{c}_{4} \sqrt{\hat{M}}\right)$ \\
& $k \simeq 705.7 \mathrm{~m}^{-1}$ & $640.7 \lesssim k \lesssim 1210.9 \mathrm{~m}^{-1}[14]$ \\
$\omega_{2}\left(\mathrm{~s}^{-1}\right)$ & $\sim 0.1$ & \\
$\frac{\omega_{2}}{v k^{2}}=\frac{\bar{\omega}_{2}}{\bar{v} \bar{k}^{2}}$ & $\ll 1$ & $\gg 10$ \\
\hline \hline
\end{tabular}

The scaling law, which states that $\bar{\omega}_{2}$ scales with the square root of $\bar{M}$, is of great practical use, as shown below.

In Eq. (3.5) two scaled material parameters appear, where $\chi_{\text {rel }}$ is a function of the magnetic field,

$$
\chi_{\text {rel }}=\frac{\left(1+\bar{\chi}_{\mathrm{H}}\right)}{\left(2+\bar{\chi}_{\mathrm{H}}\right)} \frac{\left(2+\bar{\chi}_{\mathrm{H}_{\mathrm{c}}}\right)}{\left(1+\bar{\chi}_{\mathrm{H}_{\mathrm{c}}}\right)},
$$

and relates the susceptibility at the field strength $H$ to the one at the critical field $H_{\mathrm{c}}$ for the Rosensweig instability. A step towards the scaling laws is the expansion of $\chi_{\text {rel }}$ in powers of the scaled distance of the magnetization to the critical value, $\hat{M}=\left(M-M_{\mathrm{c}, \infty}\right) / M_{\mathrm{c}, \infty}$,

$$
\chi_{\text {rel }}=1+a_{\chi} \hat{M}+b_{\chi} \hat{M}^{2} .
$$

In the following we utilize this simplified description of $\chi_{\text {rel }}$.

By expanding $\bar{M}, \bar{\omega}_{2}$, and $\bar{k}$ with respect to $\hat{M}$ around their critical values at the onset of the instability too,

$$
\begin{aligned}
\bar{M}= & 1+\hat{M}, \\
\bar{\omega}_{2}= & 0+\hat{\omega}_{2, \mathrm{~m}}=\alpha \hat{M}+\tilde{\beta} \hat{M}^{2}+\tilde{\gamma} \hat{M}^{3}+\Theta \hat{M}^{4}+\iota \hat{M}^{5} \\
& + \text { higher order terms, } \\
\bar{k}= & 1+\hat{k}_{\mathrm{m}}=1+\tilde{\lambda} \hat{M}+\delta \hat{M}^{2}+\epsilon \hat{M}^{3}+\xi \hat{M}^{4}+o \hat{M}^{5} \\
& + \text { higher order terms, }
\end{aligned}
$$

and following the procedure outlined in Ref. [20], one obtains two scaling laws valid up to a scaled magnetization of $\hat{M} \leqslant \bar{v}^{2} / 6$ :

$$
\begin{aligned}
\hat{\omega}_{2, \mathrm{~m}}= & \left(\frac{2+a_{\chi}}{\bar{v}}\right) \hat{M}+\left(\frac{1+2 a_{\chi}+b_{\chi}}{\bar{v}}-\frac{3\left(2+a_{\chi}\right)^{2}}{4 \bar{v}^{3}}\right) \hat{M}^{2} \\
& +\left(\frac{a_{\chi}+2 b_{\chi}}{\bar{v}}-\frac{3\left(2+a_{\chi}\right)\left(1+2 a_{\chi}+b_{\chi}\right)}{2 \bar{v}^{3}}\right. \\
& \left.+\frac{5\left(2+a_{\chi}\right)^{3}}{4 \bar{v}^{5}}\right) \hat{M}^{3}+\Theta \hat{M}^{4}+\iota \hat{M}^{5}, \\
\hat{k}_{\mathrm{m}}= & \left(\frac{3\left(2+a_{\chi}\right)^{2}}{2 \bar{v}^{2}}\right) \hat{M}^{2}+\left(\frac{3\left(2+a_{\chi}\right)\left(1+2 a_{\chi}+b_{\chi}\right)}{\bar{v}^{2}}\right. \\
& \left.-\frac{11\left(2+a_{\chi}\right)^{3}}{4 \bar{v}^{4}}\right) \hat{M}^{3}+\xi \hat{M}^{4}+o \hat{M}^{5} .
\end{aligned}
$$

Due to their length, the coefficients $\Theta, \xi, \iota$, and $o$ are given in Appendix A. Both scaling laws show the explicit dependence 


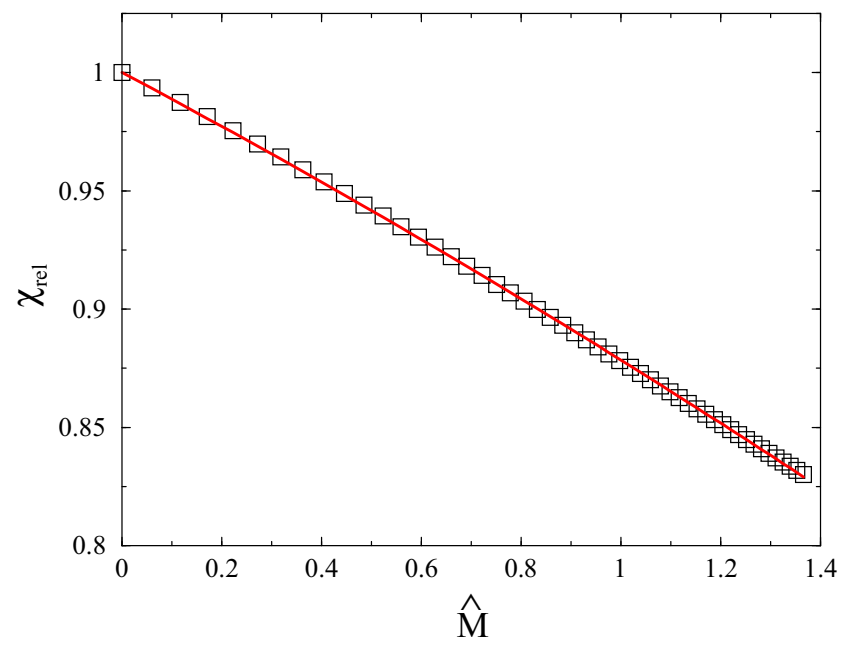

FIG. 11. The relative susceptibility $\chi_{\text {rel }}$ as function of the rescaled magnetization $\hat{M}$ of the measured curve $(\square)$ and from the secondorder approximation (3.12) (solid red line).

on the parameters viscosity and magnetization which can be any nonlinear function of $H$. Therefore Eqs. (3.14) and (3.15) represent the generalization of the results for a linear law of magnetization, i.e., for $a_{\chi}=b_{\chi}=0$, given in Ref. [20].

To prove the quality of the simplified description of $\chi_{\text {rel }}$ by Eq. (3.12), in Fig. 11 the experimental values of $\chi_{\text {rel }}$ based on the magnetization curve shown in Fig. 3 are determined by fitting that magnetization by the model proposed in Ref. [23]. The solid line represents Eq. (3.12) with $a_{\chi}=-0.1118$ and $b_{\chi}=-0.0097$, resulting in a very good agreement with the experimental data.

\section{B. The finite layer approximation for a highly viscous fluids}

It is known from a previous study of less viscous fluids [13] that a layer thickness of about the critical wavelength $\lambda_{c}$ is necessary to represent the case $h \rightarrow \infty$ for the maximal growth rate as well as for the corresponding wave number, as shown in Figs. 12(a) and 12(b).

That rule is no longer valid for more viscous fluids like APG E32 $\left(\lambda_{\mathrm{c}} \simeq 11 \mathrm{~mm}\right)$ as Fig. 12(d) displays. The results for the wave number deviate considerably from the results of the scaling law; compare the long-dashed black line $(h=$ $9 \mathrm{~mm}$ ) and the red filled circles in Fig. 12(d). By choosing a layer thickness of $h=15 \mathrm{~mm}$, the results stemming from the numerical solution of the dispersion relation (3.1) agree rather well with the data from the scaling laws; compare solid cyan lines and filled red circles in Fig. 12(d). Note that the maximal growth rate is not sensitive to $h$, as shown in Fig. 12(c). In summary, the rule can be formulated that for magnetic fluids with high viscosities a larger filling depth than in the case of low viscosities has to be used, in order to approximate the results of $h \rightarrow \infty$.

\section{RESULTS AND DISCUSSION}

We will next compare the experimentally determined growth rates with the calculated ones for our particular fluid (Sec. IV A). Then we widen our scope and compare as well the decay rates with the model (Sec. IV B). Eventually some deviations are discussed in the context of structured ferrofluids (Sec. IV C).

\section{A. Comparing the growth rate in experiment and theory}

For our kind of magnetic fluids it was argued in the Introduction that their high viscosity paves the way into a scaling regime hitherto not accessible. That claim is now proven since a value of 56.4 for the upper bound $\bar{v}^{2} / 6$ of the scaling regime results, as summarized in Tables III. That means that for experimentally feasible scaled supercritical magnetizations $\hat{M}$ the region $\hat{M} \leqslant \bar{v}^{2} / 6$ is approachable. The corresponding Eq. (3.14) for the maximal growth rate states that $\hat{\omega}_{2, \mathrm{~m}}$ should increase mainly linearly with $\hat{M}$ as long as $\hat{M}$ is not too large.

To confirm this scaling behavior, the experimentally determined growth rates from Fig. 10 are plotted together with a Levenberg-Marquard fit [41] of the maximal growth rate obtained from Eq. (3.14) versus the the magnetization $M$ as shown in Fig. 13. The agreement between the two data sets is convincing. In Table IV we present in line 1 the parameters for viscosity and surface tension, obtained from the fit. For comparison, we reprint in line 0 the measured values. The fitted surface tension is well within the error bars of the measured value, whereas the fitted viscosity is only $6 \%$ below the measured one. Thus the theoretically predicted linear dependence of the growth rates on $M$ is experimentally confirmed.

\section{B. Comparing growth and decay}

Next we focus as well on the experimental data for the decay, which are plotted together with the growth data in Fig. 14. The decay rates $(\bullet)$ are scattering more widely in comparison to the growth rates $(\boldsymbol{\square})$. This may be due to the fact that the decay rates could not be resolved in the bistability range, and thus not in the immediate vicinity of $M_{\mathrm{c}}$, in contrast to the growth rates. The black dashed line marks the outcome of a fit of Eq. (3.14) to all experimental values. Also in this extended range the fit describes the measured growth and decay rates to some extent. In Table IV we present in line 2 the fit parameters for viscosity and surface tension. The fitted surface tension is well within the error bars of the measured value, whereas the fitted viscosity is about $20 \%$ above the measured one.

Most importantly, inspecting the measured data more closely, one observes a different inclination for growth and decay rates with respect to $M$. Obviously this systematic deviation is not matched by Eq. (3.14). As a possible origin of the different inclinations, one may suspect that utilizing the static surface tension in Eq. (3.14) is not a sufficient approximation. Indeed during the growth of the peaks new surface area is generated, and the diffusion of surfactants from the bulk of the ferrofluid towards the surface may lag behind. Similarly, during the decay of the peaks surface area is annihilated, and the surface density of surfactants may there exceed the equilibrium concentration. Therefore we adopt a growth-rate-dependent dynamic surface tension according to

$$
\sigma\left(\omega_{2}\right)=\sigma_{\mathrm{S}}+\omega_{2} \varsigma
$$



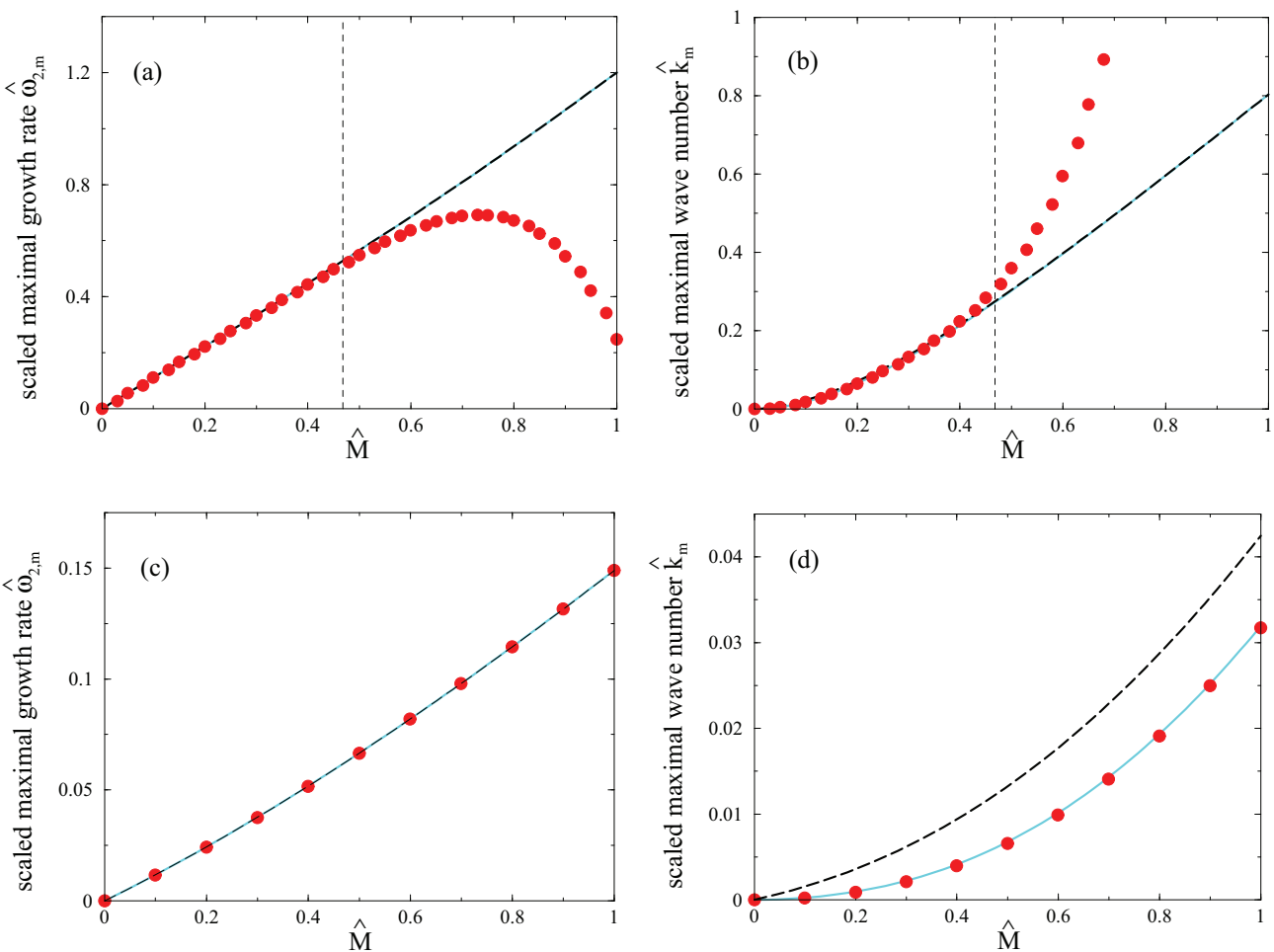

FIG. 12. The scaled maximal growth rate $\hat{\omega}_{2, \mathrm{~m}}$ [panels (a) and (c)] and the scaled maximal wave number $\hat{k}_{\mathrm{m}}[(\mathrm{b}),(\mathrm{d})]$ against the rescaled magnetization for a magnetic fluid like APG E32 but with a tenth of the original dynamical viscosity [(a),(b)] and for APG E32 itself [(c),(d)]. The solid cyan (long-dashed black) line indicates the numerical data of the dispersion relation (3.1) for $h=15$ (9) $\mathrm{mm}$, the filled red circles the results of the scaling laws. The thin vertical dashed black lines in (a) and (b) display $\bar{v}^{2} / 6$, the limit of the validity of the scaling laws (3.14) and (3.15).

where $\sigma_{\mathrm{S}}$ denotes the static surface tension and $\varsigma$ a coefficient of dimension $\mathrm{N}(\mathrm{ms})^{-1}$. In Fig. 14 the orange dashed line marks the outcome of the fit. It follows the black dashed line, and thus cannot explain the different inclinations.

In a next attempt to describe the different inclinations we postulate a growth-rate-dependent viscosity in the form of

$$
\eta(\omega)=\eta_{\mathrm{S}}+\omega_{2} \vartheta
$$

where $\vartheta$ is a coefficient of dimension Pa $\mathrm{s}^{2}$. In Fig. 14 a fit by Eq. (4.2) is marked by the solid blue line. Obviously this phenomenological ansatz meets the data remarkably well.

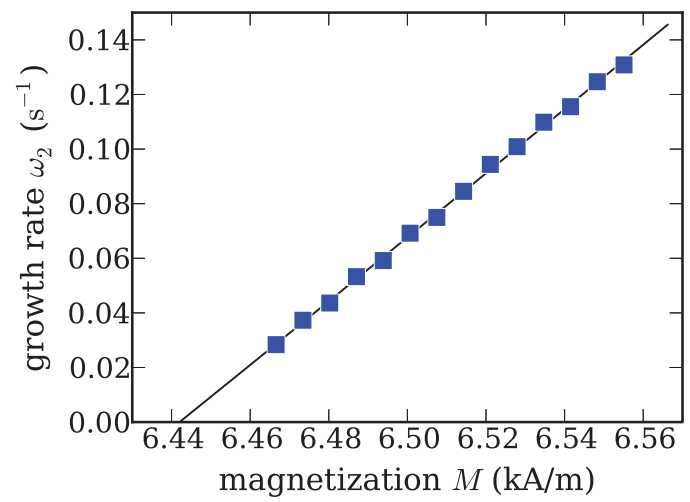

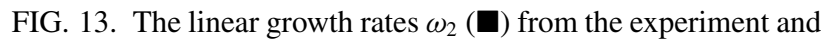
the maximal growth rate $\omega_{2, \mathrm{~m}}$ (solid black line) from theory as a function of the magnetization $M$.

\section{Discussion of deviations}

A possible explanation of this complex behavior is based upon the formation of chains of magnetic particles, which is indicated by the enhanced shear thinning as recorded in

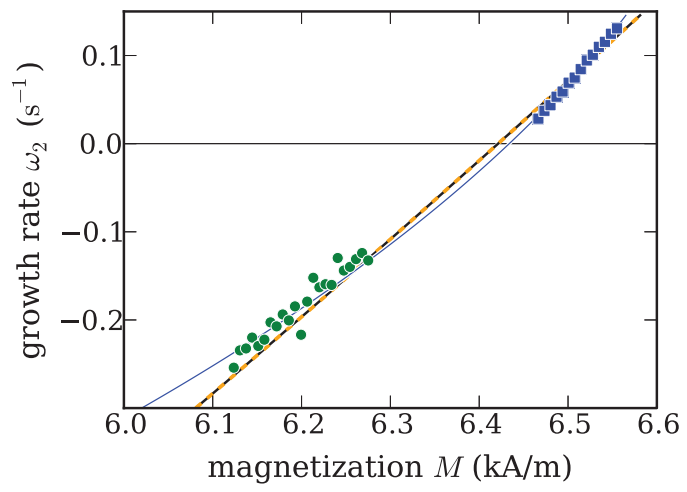

FIG. 14. The growth $(\mathbf{\square})$ and decay $(\bullet)$ rates $\omega_{2}$ of the pattern amplitude as a function of the magnetization $M$. The symbols represent the measured data. The black dashed line shows a fit of Eq. (3.14) to the experimental growth and decay rates, with the parameters given in line 2 of Table IV. The orange dashed line marks as well a fit by Eq. (3.14), but is taking into account a growth-rate-dependent surface tension, as described by Eq. (4.1). For the parameters see line 3 of Table IV. The solid blue line displays a fit taking into account a growth-rate-dependent viscosity according to Eq. (4.2). For parameters see line 4 of Table IV. 
TABLE IV. Results obtained for fitting Eq. (3.14) to the experimental data. The filling depth was $h=5$ mm, and the wave number of maximal growth was fixed to $k=544.44 \mathrm{~m}^{-1}$, as determined from the experiment; see Fig. 7. The symbol 1 marks a fit of growth data only, whereas $1 \mathrm{~L}$ indicates that growth and decay data were taken into account.

\begin{tabular}{|c|c|c|c|c|c|c|c|c|}
\hline & Direction & Property & $\eta_{\mathrm{S}}(\mathrm{Pa} \mathrm{s})$ & $\vartheta\left(\frac{\mathrm{Pas}^{2}}{\mathrm{rad}}\right)$ & $\sigma_{\mathrm{S}}\left(\frac{\mathrm{mN}}{\mathrm{m}}\right)$ & $\varsigma\left(\frac{\mathrm{mN}}{\mathrm{ms}}\right)$ & $M_{\mathrm{c}}\left(\frac{\mathrm{kA}}{\mathrm{m}}\right)$ & $B_{\mathrm{c}}(\mathrm{mT})$ \\
\hline 0 & & measured parameters & $4.48 \pm 0.2$ & & $30.9 \pm 5$ & & & \\
\hline 1 & 1 & all static parameters & 4.2 & 0 & 34.3 & 0 & 6.442 & 11.24 \\
\hline 2 & 11 & all static parameters & 5.5 & 0 & 33.8 & 0 & 6.421 & 11.20 \\
\hline 3 & 11 & dynamic surface tension & 5.5 & 0 & 33.8 & -9.9 & 6.421 & 11.20 \\
\hline 4 & 11 & non-Newtonian viscosity & 5.2 & -4.8 & 34.1 & 0 & 6.434 & 11.22 \\
\hline
\end{tabular}

Fig. 5. The chain formation will be most prominent in the higher magnetic field in the spikes at the starting amplitude $A_{\text {high }}$, marked in Fig. 6. These chains then increase the magnetoviscosity during the decay of the spikes, which retards the decay (cf. paths $\downarrow 2$ and $\downarrow 3$ b in Fig. 6). During the decay they are partially destroyed. As a consequence, after switching again to an overcritical induction, the growth of the spikes (path $\uparrow 3 a$ ) is comparatively faster. In contrast, our theory is based on Newtonian fluids. An extension to shear thinning and structured liquids has not yet been developed.

We are next comparing the critical inductions in the last column of Table IV. The static fit of the growth process yields $B_{\mathrm{c} 1}=11.24 \mathrm{mT}$ and deviates by only $1 \%$ from the mean value $\bar{B}_{\mathrm{c}}=11.36 \mathrm{mT}$ obtained by a fit of the full dynamics by means of amplitude equations in Ref. [18]. All other values for $B_{\mathrm{c}}$ underestimate this value slightly more (cf. lines $2-4$ ). In the latter three cases the growth and decay were taken into account. This is a confirmation that foremost the decay is affected by chain formation in the spikes.

We now discuss four further effects which may have impact on our experiment.

First, the experiments are performed in a finite container which comprises only 27 spikes on a hexagonal lattice, whereas the theory considers a laterally infinite layer. Our finite circular size does indeed suppress the onset of a hexagonal pattern, due to the ramp described above.

Second, by seeding a regular hexagonal pattern at large amplitude the selected wavelength may differ from the wavelength of maximal growth. This can in principle shift the experimental threshold towards higher values. However, it was demonstrated by linear stability analysis that this effect can be neglected in the limit of high viscosities [38].

Third, magnetophoresis may take place in the crests of the pattern, creating an inhomogeneous distribution of magnetite. Even though the timescale for separation in a low viscosity MF is in days [37,42] and our measurements last only hours, an effect cannot be completely excluded.

A fourth reason may be that instead of the shear viscosity the extensional viscosity has to be taken into account in Eq.(3.1). Indeed, besides a small viscous sublayer, the flow profile of surface waves can "be described by a potential and is rotational free and purely elongational" [43]. Most recently a capillary breakup extensional rheometer was subjected to magnetic fields oriented along the direction of the capillary [44]. For increasing fields an enlarged elongational viscosity was observed. This effect was also attributed to chain formation. However, to measure the extensional viscosity of ferrofluids is a difficult task, and sensitive devices have not yet been developed.

\section{CONCLUSION}

Using a highly viscous magnetic fluid, the dynamics of the formation of the Rosensweig instability can be slowed down to the order of minutes. Therefore, it is possible to measure the dynamics using a two-dimensional imaging technique, in contrast to previous work [14] where only a one-dimensional cut through the two-dimensional pattern was accomplished. By means of a specific measurement protocol we were able to seed regular patterns of small amplitude, suitable for a comparison with linear theory. From the evolution of their amplitudes we could estimate the linear growth and decay rates, respectively. Our experiment confirmed a linear scaling of the growth rate with the magnetic inductions, as predicted [20] for the immediate vicinity of the bifurcation point. Thus the scaling behavior of the growth rate is now confirmed for supercritical magnetizations not only above [14] but also below the boundary of the two scaling regimes at $\bar{v}^{2} / 6$.

Additionally, we uncovered that the rates of growth and decay are slightly different, a phenomenon not predicted by the theory. A possible origin of this discrepancy is the formation of chains of magnetic particles. Their presence in our ferrofluid is indicated by the magnetically enhanced shear thinning. The buildup of chains in the static spikes, and their subsequent destruction during the decay may change the effective viscosity of the structured ferrofluid, and thus explain the deviations.

So far our theory is based on Newtonian liquids. An extension to shear thinning and structured ferrofluids is left to future investigations. It may be able to reproduce the scaling of the effective viscosity as described phenomenologically by Eq. (3.6c).

\section{ACKNOWLEDGMENTS}

We thank M. Märkl for measuring the surface tension of the magnetic fluid used, and Tobias Lang for recording the photo in Fig. 2(c). The temperature-controlled container was made with the help of Klaus Oetter and the mechanical and electronic workshop the university of Bayreuth. Moreover discussions with Thomas Friedrich, Werner Köhler, Konstantin Morozov, and Christian Wagner are gratefully acknowledged. R.R. is deeply indebted to the Emil-Warburg foundation for financially supporting repair and upgrade of the magnetorheometer. 


\section{APPENDIX}

The coefficients for the fourth and fifth order of $\hat{M}$ in the scaling laws (3.14) and (3.15) are

$$
\begin{gathered}
\Theta=\frac{b_{\chi}}{\bar{v}}-\frac{6 a_{\chi}\left(4+3 a_{\chi}\right)+3 b_{\chi}\left(8 a_{\chi}+b_{\chi}+10\right)+3}{4 \bar{\nu}^{3}}+\frac{3\left(2+a_{\chi}\right)^{2}\left(3 a_{\chi}^{2}+32 a_{\chi}+22+10 b_{\chi}\right)}{\bar{v}^{5}}-\frac{85\left(2+a_{\chi}\right)^{4}}{32 \bar{v}^{7}}, \\
\xi=\frac{6 a_{\chi}\left(4+3 a_{\chi}\right)+3 b_{\chi}\left(8 a_{\chi}+b_{\chi}+10\right)+3}{2 \bar{v}^{2}}-\frac{3\left(2+a_{\chi}\right)^{2}\left(9 a_{\chi}^{2}+80 a_{\chi}+58+22 b_{\chi}\right)}{8 \bar{v}^{4}}+\frac{99\left(2+a_{\chi}\right)^{4}}{\bar{v}^{6}} \\
\iota=-\frac{3 a_{\chi}\left(2 a_{\chi}+1\right)+6 b_{\chi}\left(3 a_{\chi}+2+b_{\chi}\right)}{2 \bar{v}^{3}}-\frac{\left(2+a_{\chi}\right)^{3}\left(93 a_{\chi}^{2}+712 a_{\chi}+170 b_{\chi}+542\right)}{16 \bar{v}^{7}} \\
\quad+\frac{3\left(2+a_{\chi}\right)\left[12 a_{\chi}^{3}+a_{\chi}^{2}\left(6 b_{\chi}+79\right)+a_{\chi}\left(54 b_{\chi}+102\right)+b_{\chi}\left(54+5 b_{\chi}\right)+29\right]}{4 \bar{v}^{5}}+\frac{407\left(2+a_{\chi}\right)^{5}}{64 \bar{v}^{9}}, \\
o=\frac{3 a_{\chi}\left(2 a_{\chi}+1\right)+6 b_{\chi}\left(3 a_{\chi}+2+b_{\chi}\right)}{\bar{v}^{2}}+\frac{3\left(2+a_{\chi}\right)^{3}\left(23 a_{\chi}^{2}+158 a_{\chi}+33 b_{\chi}+125\right)}{\bar{v}^{6}} \\
-\frac{3\left(2+a_{\chi}\right)\left[36 a_{\chi}^{3}+a_{\chi}^{2}\left(18 b_{\chi}+217\right)+a_{\chi}\left(138 b_{\chi}+282\right)+b_{\chi}\left(138+11 b_{\chi}\right)+83\right]}{4 \bar{v}^{4}}-\frac{491\left(2+a_{\chi}\right)^{5}}{32 \bar{v}^{8}} .
\end{gathered}
$$

[1] R. Edgeworth, B. J. Dalton, and T. Parnell, Eur. J. Phys. 5, 198 (1984).

[2] J. Eggers, Rev. Mod. Phys. 69, 865 (1997).

[3] A. Rothert, R. Richter, and I. Rehberg, Phys. Rev. Lett. 87, 084501 (2001).

[4] Univ. of Queensland, The pitch drop experiment, http://smp.uq. edu.au/content/pitch-drop-experiment (26 April 2013).

[5] http://www.thetenthwatch.com (13 February 2015).

[6] R. Richter, Europhys. News 42, 17 (2011).

[7] D. Castellvecchi, Phys. Rev. Focus 15, 18 (2005).

[8] M. D. Cowley and R. E. Rosensweig, J. Fluid Mech. 30, 671 (1967).

[9] R. E. Rosensweig, Ferrohydrodynamics (Cambridge University Press, Cambridge, 1985).

[10] D. Salin, Europhys. Lett. 21, 667 (1993).

[11] J. Weilepp and H. R. Brand, J. Phys. II (France) 6, 419 (1996).

[12] A. Lange, B. Reimann, and R. Richter, Phys. Rev. E 61, 5528 (2000).

[13] A. Lange, B. Reimann, and R. Richter, Magnetohydrodynamics 37, 261 (2001).

[14] H. Knieling, R. Richter, I. Rehberg, G. Matthies, and A. Lange, Phys. Rev. E 76, 066301 (2007).

[15] C. Gollwitzer, I. Rehberg, A. Lange, and R. Richter, Frozensweig: A cool instability in the limit $\eta \rightarrow \infty$, in Book of abstracts of the 9th German Ferrofluid Workshop, Benediktbeuern, 2009 (unpublished).

[16] R. Richter and J. Bläsing, Rev. Sci. Instrum. 72, 1729 (2001).

[17] C. Gollwitzer, G. Matthies, R. Richter, I. Rehberg, and L. Tobiska, J. Fluid Mech. 571, 455 (2007).

[18] C. Gollwitzer, I. Rehberg, and R. Richter, New J. Phys. 12, 093037 (2010)

[19] D. J. B. Lloyd, C. Gollwitzer, I. Rehberg, and R. Richter, J. Fluid Mech. 783, 283 (2015).

[20] A. Lange, Europhys. Lett. 55, 327 (2001).
[21] H. Knieling, I. Rehberg, and R. Richter, in 12th International Conference on Magnetic Fluids, edited by H. Yamaguchi, Physics Procedia Vol. 9 (Elsevier, Amsterdam, 2010), pp. 199-204.

[22] C. Gollwitzer, M. Krekhova, G. Lattermann, I. Rehberg, and R. Richter, Soft Matter 5, 2093 (2009).

[23] A. O. Ivanov and O. B. Kuznetsova, Phys. Rev. E 64, 041405 (2001).

[24] M. I. Shliomis, Sov. Phys. JETP 34, 1291 (1972).

[25] R. E. Rosensweig, J. Appl. Phys. 57, 4259 (1985).

[26] R. E. Zelazo and J. R. Melcher, J. Fluid Mech. 39, 1 (1969).

[27] J. Rault, J. Non-Cryst. Solids 271, 177 (2000).

[28] R. I. Tanner, Engineering Rheology (Oxford University Press, Oxford, 2000).

[29] A. W. Sisko, Ind. Eng Chem. 50, 1789 (1958).

[30] M. I. Shliomis, Usp. Fiz. Nauk 112, 427 (1974) [Sov. Phys. Usp. 17, 153 (1974)].

[31] M. Klokkenburg, R. P. A. Dullens, W. K. Kegel, B. H. Erne, and A. P. Philipse, Phys. Rev. Lett. 96, 037203 (2006).

[32] S. Odenbach and H. Störk, J. Magn. Magn. Mater. 183, 188 (1998).

[33] Colloidal Magnetic Fluids: Basics, Development and Applications of Ferrofluids, edited by S. Odenbach, Lecture Notes in Physics Vol. 763 (Springer, Berlin, 2009).

[34] Y. Cao and Z. Ding, J. Magn. Magn. Mater. 355, 93 (2014).

[35] C. Gollwitzer, I. Rehberg, and R. Richter, J. Phys.: Condens. Matter 18, S2643 (2006).

[36] T. Friedrich, A. Lange, I. Rehberg, and R. Richter, Magnetohydrodynamics 47, 167 (2011).

[37] O. Lavrova, V. Polevikov, and L. Tobiska, Math. Model. Anal. 15, 223 (2010).

[38] B. Reimann, R. Richter, I. Rehberg, and A. Lange, Phys. Rev. E 68, 036220 (2003).

[39] A. Lange, Magnetohydrodynamics 39, 65 (2003). 
[40] Due to the equivalence of $\hat{B}$ and $\hat{M}$, the scaling law $\hat{k}_{\mathrm{m}}=c_{3} \hat{B}+$ $c_{4} \sqrt{\hat{B}}$ in [20] is adapted to $\hat{k}_{\mathrm{m}}=\tilde{c}_{3} \hat{M}+\tilde{c}_{4} \sqrt{\hat{M}}$.

[41] H. Press, A. Teukolsky, T. Vetterling, and P. Flannery, Numerical Recipes in C++. The Art of Computer Programming (Cambridge University Press, New York, 2002).
[42] A. S. Ivanov and A. F. Pshenichnikov, J. Magn. Magn. Mater. 322, 2575 (2010).

[43] A. Kityk and C. Wagner, Europhys. Lett. 75, 441 (2006).

[44] F. J. Galindo-Rosales, J. P. Segovia-Gutiérrez, F. T. Pinho, M. A. Alves, and J. de Vicente, J. Rheol. 59, 193 (2015). 